\title{
General Results for the Transmuted Family of Distributions and New Models
}

\author{
Marcelo Bourguignon, ${ }^{1}$ Indranil Ghosh, ${ }^{2}$ and Gauss M. Cordeiro ${ }^{3}$ \\ ${ }^{1}$ Departamento de Estatística, Universidade Federal do Rio Grande do Norte, 59078-970 Natal, RN, Brazil \\ ${ }^{2}$ Department of Mathematics and Statistics, University of North Carolina Wilmington, Wilmington, NC, USA \\ ${ }^{3}$ Departamento de Estatística, Universidade Federal de Pernambuco, 50740-540 Recife, PE, Brazil
}

Correspondence should be addressed to Marcelo Bourguignon; m.p.bourguignon@gmail.com

Received 4 October 2015; Accepted 29 December 2015

Academic Editor: Zacharias Psaradakis

Copyright (c) 2016 Marcelo Bourguignon et al. This is an open access article distributed under the Creative Commons Attribution License, which permits unrestricted use, distribution, and reproduction in any medium, provided the original work is properly cited.

\begin{abstract}
The transmuted family of distributions has been receiving increased attention over the last few years. For a baseline $G$ distribution, we derive a simple representation for the transmuted- $G$ family density function as a linear mixture of the $G$ and exponentiated$G$ densities. We investigate the asymptotes and shapes and obtain explicit expressions for the ordinary and incomplete moments, quantile and generating functions, mean deviations, Rényi and Shannon entropies, and order statistics and their moments. We estimate the model parameters of the family by the method of maximum likelihood. We prove empirically the flexibility of the proposed model by means of an application to a real data set.
\end{abstract}

\section{Introduction}

Adding parameters to a well-established distribution is a time honored device for obtaining more flexible new families of distributions. Shaw and Buckley [1] pioneered an interesting method of adding a new parameter to an existing distribution that would offer more distributional flexibility. They used the quadratic rank transmutation map (QRTM) in order to generate a flexible family of distributions. The generated family, also called the transmuted extended distribution, includes the parent distribution as a special case and gives more flexibility to model various types of data.

In the last three years, there has been a growing interest in transmuted distributions and several of them have been investigated. A significant amount of work has been attributed towards developing a new transmuted model and subsequently discussing its utilities as enhanced flexibility in modeling various types of real life data, where the parent model does not provide a good fit. Aryal and Tsokos [2] defined the transmuted generalized extreme value distribution and studied some basic mathematical characteristics of the transmuted Gumbel distribution and its applications to climate data. Aryal and Tsokos [3] presented a new generalized Weibull distribution called the transmuted Weibull distribution. Recently, Aryal [4] proposed and studied various structural properties of the transmuted log-logistic distribution. Khan and King [5] introduced the transmuted modified Weibull distribution, which extends the transmuted Weibull distribution [3], and studied its mathematical properties and maximum likelihood estimation of the model parameters. Elbatal [6] proposed the transmuted modified inverse Weibull distribution. Elbatal and Aryal [7] explored the transmuted additive Weibull model, which extends the additive Weibull distribution and some other distributions using the QRTM method [1]. However, several published works did not investigate many properties such as finite mixture of the density function, Rényi and Shannon entropies, extreme values, probability weighted moments (PWMs), and bivariate and multivariate generalization. This paper aims to fill out this gap in the existing literature and contribute with general properties of the transmuted family.

This vast amount of literature merits for a detailed study for the most general transmuted family of distributions, which is our major motivation to carry out this work. In this paper, we derive general mathematical properties for the transmuted family, which hold for any baseline distribution, such as the ordinary, central, and incomplete moments, quantile and generating functions, mean deviations, Rényi and 
Shannon entropies, extreme values, PWMs, order statistics and their moments, and bivariate and multivariate generalizations. We provide a comprehensive description of these properties with the hope that the transmuted family will attract wider applications in biology, medicine, economics, reliability, and engineering and in other areas of research. We also introduce new distributions based on the transmuted construction.

The rest of the paper is organized as follows. In Section 2, we discuss the general theory behind the transmuted distribution and present useful representations for the density and cumulative functions. In Section 3, we investigate its asymptotes and shapes. In Section 4, we provide an algorithm for generating samples from the transmuted family based on its quantile function (qf). In Section 5, we derive expressions for the moments and generating function. In Section 6, we obtain mean deviations and provide some examples. In Section 7, we present two special transmuted models. In Section 8, we discuss the limiting behavior of the extreme statistics. In Section 9, we derive the PWMs. In Section 10, we obtain the order statistics. We derive expressions for the Shannon and Rényi entropies and Kullback-Leibler divergence measure in Section 11. We introduce in Section 12 the bivariate and multivariate extensions of the univariate transmuted family. In Section 13, we use the maximum likelihood method to estimate the model parameters. In Section 14, we fit some special models of the transmuted family to a real data set to prove empirically its usefulness. In Section 15, we offer some concluding remarks.

\section{Distribution and Density Functions}

Let $F_{1}$ and $F_{2}$ be the cumulative distribution functions (cdfs) of two models with a common sample space. The general rank transmutation as given in Shaw and Buckley [1] is defined as $G_{R_{12}}(u)=F_{2}\left(F_{1}^{-1}(u)\right)$ and $G_{R_{21}}(u)=F_{1}\left(F_{2}^{-1}(u)\right)$. Note that the qf is defined by $F^{-1}(y)=\inf _{x \in \mathbb{R}}\{F(x) \geq y\}$ for $y \in[0,1]$. Functions $G_{R_{12}}(u)$ and $G_{R_{21}}(u)$ both map the unit interval $I=[0,1]$ into itself and, under suitable assumptions, are mutual inverses and satisfy $G_{R_{i j}}(0)=0$ and $G_{R_{i j}}(1)=1$ (for $i=1,2$ ). The QRTM is defined by $G_{R_{12}}(u)=u+$ $\lambda u(1-u),|\lambda| \leq 1$, from which it follows that $F_{2}(x)=$ $(1+\lambda) F_{1}(x)-\lambda F_{1}(x)^{2}$. Differentiating gives $f_{2}(x)=f_{1}(x)[1+$ $\lambda-2 \lambda F_{1}(x)$, where $f_{1}(x)$ and $f_{2}(x)$ are the probability density functions (pdfs) corresponding to the cdfs $F_{1}(x)$ and $F_{2}(x)$, respectively. For more details about the QRTM approach, see Shaw and Buckley [1].

A random variable $X$ has the transmuted-G $(T G)$ family if the pdf and cdf are defined through the QRTM method by (for $\lambda \in[-1,1]$ )

$$
\begin{aligned}
& f(x)=f(x ; \xi, \lambda)=[1+\lambda-2 \lambda G(x ; \xi)] g(x ; \xi), \\
& x \in \mathscr{D} \subseteq \mathbb{R}, \\
& F(x)=F(x ; \xi, \lambda)=(1+\lambda) G(x ; \xi)-\lambda G(x ; \xi)^{2},
\end{aligned}
$$

where $G(x ; \xi)$ is the parent cdf and $g(x ; \xi)$ is the parent pdf. Both functions depend on the parameter vector $\xi$. For $\lambda=0$, it reduces to the parent model. Hereafter, the random variable $X$ following (1) with parameter $\lambda$ and baseline vector of parameters $\xi$ is denoted by $X \sim T G(\lambda, \xi)$. The computations for fitting family (1) to real data in practical problems can be easily performed using the AdequacyModel script in the $R$ software.

For an arbitrary baseline $\operatorname{cdf} G(x ; \xi)$, a random variable is said to have the $\operatorname{Exp}-G$ distribution with power parameter $\alpha>0$, say $Y \sim \operatorname{Exp}-G(\xi ; \alpha)$, if its pdf and cdf are given by

$$
\begin{aligned}
& \pi(x ; \xi, \alpha)=\alpha G(x ; \xi)^{\alpha-1} g(x ; \xi), \\
& \Pi(x ; \xi, \alpha)=G(x ; \xi)^{\alpha},
\end{aligned}
$$

respectively. Note that $\pi(x ; \xi, 1)=g(x ; \xi)$. The properties of exponentiated distributions have been studied by many authors in recent years. See, for example, Mudholkar and Srivastava [8] for exponentiated Weibull, Gupta et al. [9] for exponentiated Pareto, Gupta and Kundu [10] for exponentiated exponential, Nadarajah [11] for exponentiated Gumbel, Kakde and Shirke [12] for exponentiated lognormal, and Nadarajah and Gupta [13] for exponentiated gamma distributions.

Theorem 1. The density function of $X$ can be expressed as the linear mixture

$$
f(x ; \xi, \lambda)=c g(x ; \xi)+(1-c) \pi(x ; \xi, 2)
$$

where $c=1+\lambda$.

Corollary 2. If $\lambda=-1$, then $X \sim \operatorname{Exp}-G(\xi ; 2)$.

Theorem 1 is important to obtain some measures of $X$ from those of exponentiated distributions. This result plays an important role in the paper, since we can obtain, for example, the moments, generating function, and mean deviations of $X$. Established explicit expressions for these measures can be simpler than using numerical integration.

The hazard rate function (hrf) of $X$ is given by

$$
\begin{aligned}
h(x ; \xi, \lambda) & =\frac{[1+\lambda-2 \lambda G(x ; \xi)] g(x)}{[1-G(x ; \xi)][1-\lambda G(x ; \xi)]} \\
& =h_{G}(x) \frac{[1+\lambda-2 \lambda G(x ; \xi)]}{1-\lambda G(x ; \xi)}, \quad x>0,
\end{aligned}
$$

where $h_{G}(x)$ is the baseline hrf. The multiplying quantity [ $1+$ $\lambda-2 \lambda G(x ; \xi)] /[1-\lambda G(x ; \xi)]$ is a kind of correction factor for the baseline hrf.

Equation (5) can deal with general situations for modeling survival data with various hrf shapes. From this equation, we note that $h(x ; \xi, \lambda) / h_{G}(x ; \xi)$ is decreasing in $x$ for $\lambda \geq 0$ and it is increasing in $x$ for $\lambda \leq 0$. Additionally, we have

$$
\begin{aligned}
h_{G}(x) & \leq h(x) \leq(1+\lambda) h_{G}(x), \\
(1+\lambda) h_{G}(t) & \leq h(x) \leq h_{G}(x),
\end{aligned}
$$

for $\lambda \geq 0$ and $\lambda \leq 0$, respectively. 
Equation (5) can be expressed as

$$
\begin{aligned}
h(x ; \boldsymbol{\xi}, \lambda)= & w(t) h_{G}(x ; \xi) \\
& +[1-w(t)] h_{\text {Exp-G }}(x ; \xi, 2),
\end{aligned}
$$

where $w(t)=\{c[1-G(x ; \xi)]\} /\left\{1-(1+\lambda) G(x ; \xi)+\lambda[G(x ; \xi)]^{2}\right\}$, $c=1+\lambda$, and $h_{G}(x ; \xi)$ and $h_{\text {Exp-G }}(x ; \xi, 2)$ are the hrfs of the $G$ and Exp- $G$ distributions, respectively.

\section{Asymptotes and Shapes}

Proposition 3. The asymptotics of (1) and (2) as $G(x) \rightarrow 0$ are

(i) $F(x) \sim(1+\lambda) G(x)$,

(ii) $f(x) \sim(1+\lambda) g(x)$,

(iii) $h(x) \sim(1+\lambda) g(x) / \bar{G}(x)$, where $\bar{G}(x)=1-G(x)$.

Proposition 4. The asymptotics of (1) and (2) as $x \rightarrow \infty$ are

(i) $1-F(x) \sim \lambda G(x)[G(x)-1]$,

(ii) $f(x) \sim(1-\lambda) g(x)$,

(iii) $h(x) \sim(1-\lambda) g(x) / \bar{G}(x)$.

The shapes of the density and hazard functions of $X$ can be described analytically. The critical points of the TG pdf are the roots of the equation

$$
\frac{g^{\prime}(x)}{g(x)}-\frac{2 \lambda g(x)}{[1+\lambda-2 \lambda G(x)]}=0 .
$$

There may be more than one root to (8). Let $\delta(x)=$ $\left(d^{2} / d x^{2}\right) \log [f(x)]$. We have

$\delta(x)$

$$
\begin{aligned}
= & \frac{g^{\prime \prime}(x) g(x)-g^{\prime}(x)^{2}}{g(x)^{2}} \\
& -\frac{2 \lambda\left[(1+\lambda) g^{\prime}(x)-2 \lambda g^{\prime}(x) G(x)+2 \lambda g(x)^{2}\right]}{(1+\lambda-2 \lambda G(x))^{2}} .
\end{aligned}
$$

If $x=x_{0}$ is a root of (9), then it corresponds to a local maximum if $\delta(x)>0$ for all $x<x_{0}$ and $\delta(x)<0$ for all $x>x_{0}$. It corresponds to a local minimum if $\delta(x)<0$ for all $x<x_{0}$ and $\delta(x)>0$ for all $x>x_{0}$. It refers to a point of inflection if either $\delta(x)>0$ for all $x \neq x_{0}$ or $\delta(x)<0$ for all $x \neq x_{0}$.

The critical points of the hrf of $X$ are obtained from

$$
\begin{aligned}
& \frac{g^{\prime}(x)}{g(x)}-\frac{2 \lambda g(x)}{[1+\lambda-2 \lambda G(x)]}+\frac{g(x)}{1-G(x)} \\
& \quad+\frac{\lambda g(x)}{1-\lambda G(x)} .
\end{aligned}
$$

Again, there may be two roots to (10). Let $\theta(x)=$ $\left(d^{2} / d x^{2}\right) \log h(x)$. We have

$$
\begin{aligned}
\theta(x)= & \frac{g^{\prime \prime}(x) g(x)-g^{\prime}(x)^{2}}{g(x)^{2}} \\
& +\frac{(1-G(x)) g^{\prime}(x)+g^{2}(x)}{[1-G(x)]^{2}} \\
& +\lambda \frac{[1-\lambda G(x)] g^{\prime}(x)+\lambda g^{2}(x)}{[1-\lambda G(x)]^{2}} \\
& -2 \lambda \frac{[1+\lambda-2 \lambda G(x)] g^{\prime}(x)+2 \lambda g^{2}(x)}{[1+\lambda-2 \lambda G(x)]^{2}} .
\end{aligned}
$$

If $x=x_{0}$ is a root of (11), then it corresponds to a local maximum if $\theta(x)>0$ for all $x<x_{0}$ and $\theta(x)<0$ for all $x>x_{0}$. It corresponds to a local minimum if $\theta(x)<0$ for all $x<x_{0}$ and $\theta(x)>0$ for all $x>x_{0}$. It refers to a point of inflection if either $\theta(x)>0$ for all $x \neq x_{0}$ or $\theta(x)<0$ for all $x \neq x_{0}$.

\section{Quantile Function and Simulation}

The qf of the TG family is given by

$$
\begin{aligned}
& Q(u ; \xi, \lambda) \\
& = \begin{cases}Q_{G}\left(\frac{1+\lambda-\sqrt{(1+\lambda)^{2}-4 \lambda u}}{2 \lambda} ; \xi\right), & \text { if } \lambda \neq 0 ; \\
Q_{G}(u ; \xi), & \text { if } \lambda=0,\end{cases}
\end{aligned}
$$

where $Q_{G}(u ; \xi)=G^{-1}(u ; \xi)$ is the inverse of the baseline cdf. The $T G$ family is easily simulated by Algorithm 1 .

Table 1 gives $Q_{G}(u ; \xi)$ and the corresponding parameters for some special distributions.

\section{Moments and Generating Function}

Many of the important characteristics and features of a distribution are determined through the ordinary moments. The $r$ th ordinary moment of $X$ is obtained from Theorem 1 as

$$
\mu_{r}^{\prime}=E\left(X^{r}\right)=c E\left(Y_{1}^{r}\right)+(1-c) E\left(Y_{2}^{r}\right),
$$

where $Y_{i} \sim \operatorname{Exp}-G(\xi ; i)$ for $i=1,2$. Some moments obtained from (13) are reported in Table 2.

The central moments $\left(\mu_{r}\right)$ and cumulants $\left(\kappa_{r}\right)$ of $X$ follow from (13) as

$$
\begin{aligned}
& \mu_{r}=\sum_{k=0}^{r}\left(\begin{array}{c}
r \\
k
\end{array}\right)(-1)^{k} \mu_{1}^{\prime k} \mu_{r-k}^{\prime}, \\
& \kappa_{r}=\mu_{r}^{\prime}-\sum_{k=1}^{r-1}\left(\begin{array}{c}
r-1 \\
k-1
\end{array}\right) \kappa_{k} \mu_{r-k}^{\prime},
\end{aligned}
$$

respectively, where $\kappa_{1}=\mu_{1}^{\prime}$. Further, the skewness and kurtosis are obtained from the third and fourth standardized cumulants $\zeta_{1}=\kappa_{3} / \kappa_{2}^{3 / 2}$ and $\zeta_{2}=\kappa_{4} / \kappa_{2}^{2}$, respectively. 
(1) Generate a random number $u$ from $U \sim U(0,1)$;

(2) If $\lambda \neq 0$ then compute a random number $x=Q_{G}\left(\left(1+\lambda-\sqrt{(1+\lambda)^{2}-4 \lambda U}\right) / 2 \lambda ; \xi\right)$; Otherwise $x=Q_{G}(U ; \xi)$;

(3) Repeat steps (1) to (2) until the required amount of random numbers to be completed.

Algorithm 1: Random number generator for the $T G$ distribution.

TABLE 1: Distributions and corresponding qfs.

\begin{tabular}{lcc}
\hline Distribution & $Q_{G}(u ; \xi)$ & $\xi$ \\
\hline Uniform $(0<x<\theta)$ & $\theta u$ & $\theta$ \\
Exponential $(x>0)$ & $\alpha^{-1} \log (1-u)$ & $\alpha$ \\
Weibull $(x>0)$ & {$\left[\alpha^{-1} \log (1-u)\right]^{1 / \beta}$} & $(\alpha, \beta)$ \\
Fréchet $(x>0)$ & $\sigma[-\log (u)]^{-1 / \lambda}$ & $(\lambda, \sigma)$ \\
Half-logistic $(x>0)$ & $-\log [(1-u) /(1+u)]$ & $\varnothing$ \\
Power function & $u^{1 / k} / \theta$ & $(\theta, k)$ \\
$(0<x<1 / \theta)$ & $\theta(1-u)^{-1 / k}$ & $(\theta, k)$ \\
Pareto $(x \geq \theta)$ & $s\left[(1-u)^{-1 / k}-1\right]^{1 / c}$ & $(s, k, c)$ \\
Burr XII $(x>0)$ & $-\log \left(u^{-1}-1\right)$ & $\varnothing$ \\
Logistic $(x \in \mathbb{R})$ & $s\left[(1-u)^{-1}-1\right]^{1 / c}$ & $(s, c)$ \\
Log-logistic $(x>0)$ & $s\left[(1-u)^{-1 / k}-1\right]$ & $(s, k)$ \\
Lomax $(x>0)$ & $\mu-\sigma \log [-\log (u)]$ & $(\mu, \sigma)$ \\
Gumbel $(x \in \mathbb{R})$ & {$\left[1-(1-u)^{1 / b}\right]^{1 / a}$} & $(a, b)$ \\
Kumaraswamy & $\Phi^{-1}((u-\mu) / \sigma)$ & $(\mu, \sigma)$ \\
$(0<x<1)$ & &
\end{tabular}

The moment generating function (mgf) of $X$, say $M(t)=$ $E\left(e^{t X}\right)$, can be expressed from Theorem 1 as

$$
M(t)=c I_{0}(\boldsymbol{\xi})+2(1-c) I_{1}(\boldsymbol{\xi})
$$

where $I_{j-1}=I_{j-1}(\xi)=\int_{0}^{1} \exp \left[t Q_{G}(u ; \xi)\right] u^{j-1} d u$ for $j=1,2$. The integrals $I_{0}(\xi)$ and $I_{1}(\xi)$ can be evaluated numerically for most parent distributions.

Three closed forms for $I_{j-1}$ (for $j=1,2$ ) follow by selecting from Table 1 the exponential (with parameter $\alpha$ ), standard logistic, and Fréchet as baseline distributions, for which $I_{j-1}=B(j, 1-\alpha / t)($ for $t>\alpha), I_{j-1}=B(j, 1-t)$ (for $t<1$ ), and $I_{j-1}=(t \sigma / \lambda+j)^{-1}$, respectively.

The characteristic function (chf) has many useful and important properties and plays a central role in statistical theory. It is particularly useful in analysis of linear combination of independent random variables. Clearly, a simple representation for the $\operatorname{chf} \phi(t)=M(i t)$ of $X$, where $i=\sqrt{-1}$, is given by

$$
\begin{aligned}
\phi(t)= & \int_{0}^{\infty} \cos (t x) f(x ; \xi, \lambda) d x \\
& +i \int_{0}^{\infty} \sin (t x) f(x ; \xi, \lambda) d x .
\end{aligned}
$$

From expansions $\cos (t x)=\sum_{r=0}^{\infty}\left((-1)^{r} /(2 r) !\right)(t x)^{2 r}$ and $\sin (t x)=\sum_{r=0}^{\infty}\left((-1)^{r} /(2 r+1) !\right)(t x)^{2 r+1}$, we obtain

$$
\phi(t)=\sum_{r=0}^{\infty} \frac{(-1)^{r} t^{2 r}}{(2 r) !} \mu_{2 r}^{\prime}+i \sum_{r=0}^{\infty} \frac{(-1)^{r} t^{2 r+1}}{(2 r+1) !} \mu_{2 r+1}^{\prime} .
$$

\section{Mean Deviations}

The $n$th incomplete moment of $X$, say $m_{n}(y)=\int_{-\infty}^{y} x^{n} f(x$; $\xi, \lambda) d x$, is expressed as

$$
m_{n}(y)=c v_{n, 1}(y)+(1-c) v_{n, 2}(y),
$$

where

$$
v_{n, j}(y)=j \int_{0}^{G(y ; \xi)} Q_{G}(u ; \xi)^{r} u^{j-1} d u \quad n=1,2 .
$$

The integral $v_{n, j}(y)$ can be determined analytically for some special models with closed form expressions for $Q_{G}(u ; \xi)$ or evaluated at least numerically for most baseline distributions. It can also be obtained for several baseline $G$ distributions using power series methods. These methods are at the heart of many aspects of applied mathematics and statistics. If this function does not have a closed form expression, it can be expressed as a power series:

$$
Q_{G}(u ; \xi)=\sum_{i=0}^{\infty} a_{i} u^{i}
$$

where coefficients $a_{i}$ are suitably chosen real numbers. For some important distributions, such as the normal, Student $t$, gamma, and beta distributions, $Q_{G}(u ; \xi)$ does not have closed form but it can be expanded as in (20). For example, for the standard normal distribution, coefficients $a_{i}^{\prime}$ s are given by

$$
a_{i}=(2 \pi)^{i / 2} \sum_{m=i}^{\infty}\left(\frac{-1}{2}\right)^{m-j}\left(\begin{array}{c}
m \\
i
\end{array}\right) p_{i}
$$

where $p_{i}=0$ (for $i=0,2,4, \ldots$ ) and $p_{i}=q_{(i-1) / 2}$ (for $i=$ $1,3,5, \ldots)$, and the $q_{k}^{\prime} \mathrm{s}$ are determined recursively from

$$
q_{k+1}=\frac{1}{2(2 k+3)} \sum_{r=0}^{k} \frac{(2 r+1)(2 k-2 r+1) q_{r} q_{k-r}}{(r+1)(2 r+1)} .
$$

Then, $q_{0}=1, q_{1}=1 / 6, q_{2}=7 / 120$, and $q_{3}=127 / 7560, \ldots$

We consider a result by Gradshteyn and Ryzhik [17] for a power series raised to a positive integer $n$ :

$$
Q_{G}(u ; \xi)^{n}=\left(\sum_{i=0}^{\infty} a_{i} u^{i}\right)^{n}=\sum_{i=0}^{\infty} c_{n, i} u^{i}
$$


TABLE 2: Distributions and their moments.

\begin{tabular}{lcc}
\hline Distribution & $\mu_{r}^{\prime}$ & Reference \\
Transmuted Weibull & $\frac{\Gamma(1+r / \beta)}{\alpha^{1 / \beta}}\left(1-\lambda+2^{-r / \beta} \lambda\right)$ & Aryal and Tsokos [3] \\
\hline Transmuted Lindley & $\frac{r !}{\theta^{r}(\theta+1)}\left[(1-\lambda)(\theta+r+1)+\frac{\lambda \theta}{2^{r-1}(\theta+1)}(2 \theta+3+r)\right]$ & Merovci [14] \\
\hline Transmuted Fréchet & $\sigma^{r} \Gamma\left(1-\frac{r}{\lambda}\right)\left[1+\lambda-2^{r / \lambda} \lambda\right], \quad r<\lambda$ & Mahmoud and Mandouh [15] \\
\hline Transmuted log-logistic & $\frac{s^{r} r \pi / c}{\sin (r \pi / c)}\left[1+\lambda-\lambda s^{c}\left(1+\frac{r}{c}\right)\right]$ & Aryal [4] \\
\hline Transmuted Pareto & $\frac{k \beta(2 k-r(1+\lambda))}{(k-r)(2 k-r)}, \quad r<k$ & Merovci and Puka [16] \\
\hline
\end{tabular}

where coefficients $c_{n, i}$ (for $i=1,2, \ldots$ ) are determined from the recurrence equation

$$
c_{n, i}=\left(i a_{0}\right)^{-1} \sum_{m=1}^{i}[m(n+1)-i] a_{m} c_{n, i-m},
$$

and $c_{n, 0}=a_{0}^{r}$. Coefficient $c_{n, i}$ can be obtained from quantities $a_{0}, \ldots, a_{i}$ in any analytical or numerical software. Hence, quantity $v_{n, j}(y)$ (for $\left.j=1,2\right)$ in (19) is given by

$$
v_{n, j}(y)=j \sum_{i=0}^{\infty} \frac{c_{n, i}}{(i+j)} G(y ; \xi)^{i+j} .
$$

An important application of the first incomplete moment of $X$ in (18) is related to the Bonferroni and Lorenz curves. These curves are very useful in economics, reliability, demography, insurance, and medicine. For a given probability $\pi$, they are given by $B(\pi)=m_{1}(q) /\left(\pi \mu_{1}^{\prime}\right)$ and $L(\pi)=m_{1}(q) / \mu_{1}^{\prime}$, where $q=Q(\pi ; \xi, \lambda)$ comes from (12).

The magnitude of dispersion associated with the population can be measured by the totality of deviations from the mean and median. Another application refers to the the deviations about the mean $\left(\delta_{1}=E\left(\left|X-\mu_{1}^{\prime}\right|\right)\right)$ and about the median $\left(\delta_{2}=E(|X-M|)\right)$ of $X$ given by

$$
\begin{aligned}
& \delta_{1}=2 \mu_{1}^{\prime} F\left(\mu_{1}^{\prime}\right)-2 m_{1}\left(\mu_{1}^{\prime}\right), \\
& \delta_{2}=\mu_{1}^{\prime}-2 m_{1}(M),
\end{aligned}
$$

respectively, where $M$ is the median of $X, \mu_{1}^{\prime}=E(X)$ is determined from (13), $F\left(\mu_{1}^{\prime}\right)$ is easily evaluated from (2), and $m_{1}(y)=c v_{1,1}(y)+(1-c) v_{1,2}(y)$ is obtained from (19) with $n=1$.

Next, we provide two applications of (19) by taking for the baseline model the exponential (with parameter $\alpha$ ) and standard logistic distributions listed in Table 1 . By using the generalized binomial expansion, we obtain $v_{1, j}(y)$ (for $j=1$ and 2) for the transmuted-exponential (TE) (with parameter $\alpha)$ and transmuted-standard logistic (TSL) as

$$
\begin{aligned}
& v_{1, j}(y)=\frac{j}{\alpha} \sum_{m=0}^{\infty} \frac{(-1)^{m}\left(1-e^{-m \alpha y}\right)}{(m+1)}\left(\begin{array}{c}
j \\
m
\end{array}\right), \\
& v_{1, j}(y)=\frac{1}{\Gamma(y)} \sum_{m=0}^{\infty} \frac{(-1)^{m} \Gamma(m+j)}{(m+1) !}\left(1-e^{-m z}\right),
\end{aligned}
$$

respectively.

\section{Special Transmuted Models}

The pdf and cdf of $X$ in (1) and (2) will be most tractable when $G(x)$ and $g(x)$ have simple analytic expressions. In this section, we present two special $T G$ models.

7.1. The Transmuted Burr XII (TBXII) Distribution. We consider the parent Burr XII distribution, where the pdf and cdf (for $x>0$ ) are $g(x)=c k s^{-c} x^{c-1}\left[1+(x / s)^{c}\right]^{-k-1}, s, k, c>0$, and $G(x)=1-\left[1+(x / s)^{c}\right]^{-k}$, respectively. Then, the TBXII density function is given by

$$
\begin{gathered}
F(x ; k, s, c, \lambda)=\left\{1-\left[1+\left(\frac{x}{s}\right)^{c}\right]^{-k}\right\} \\
\cdot\left\{1+\lambda-\lambda\left\{1-\left[1+\left(\frac{x}{s}\right)^{c}\right]^{-k}\right\}\right\},
\end{gathered}
$$

where $\lambda \in[-1,1]$. The corresponding pdf is given by

$$
\begin{aligned}
f(x ; k, s, c, \lambda)= & \frac{k c}{s}\left(\frac{x}{s}\right)^{c-1}\left[1+\left(\frac{x}{s}\right)^{c}\right]^{-k-1} \\
& \cdot\left(1-\lambda+2 \lambda\left[1+\left(\frac{x}{s}\right)^{c}\right]^{-k}\right) .
\end{aligned}
$$

The TBXII distribution includes an important special case when $k=1$ : the transmuted-log-logistic [4] distribution. Further, we obtain the transmuted Lomax [18] distribution when $c=1$. Some plots of the TBXII density function are displayed in Figure 1.

The $r$ th ordinary moment of the TBXII model can be obtained from (13) as

$$
\begin{aligned}
E\left(X^{r}\right)= & (1-\lambda) B\left(k-r c^{-1}, r c^{-1}+1\right) \\
& +\lambda B\left(2 k-r c^{-1}, r c^{-1}+1\right), \quad c k>r,
\end{aligned}
$$

where $B(\cdot, \cdot)$ is the beta function.

7.2. The Transmuted Kumaraswamy (TKw) Distribution. The baseline Kumaraswamy $(\mathrm{Kw})$ distribution has pdf and cdf, for $x \in(0,1)$ and $a, b>0$, given by $g(x)=a b x^{a-1}\left(1-x^{a}\right)^{b-1}$ and 


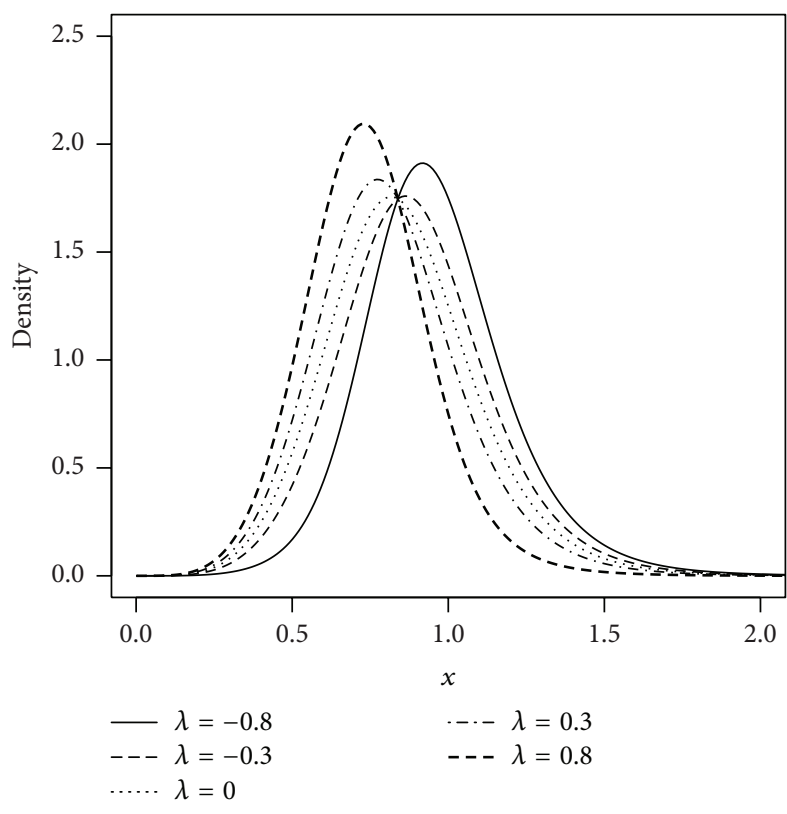

(a) $k=2, c=5$

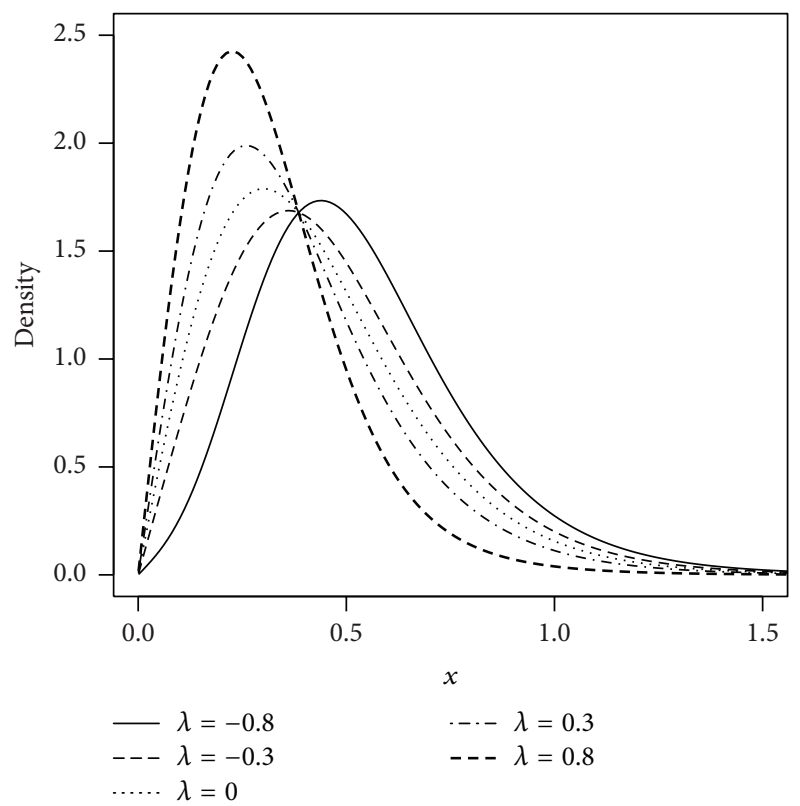

(b) $k=5, c=2$

FIGURE 1: Plots of the TBXII density function for some parameter values.

$G(x)=1-\left(1-x^{a}\right)^{b}$, respectively. Then, the TKw cdf is given by

$$
F(x ; a, b, \lambda)=\left[1-\left(1-x^{a}\right)^{b}\right]\left[1+\lambda\left(1-x^{a}\right)^{b}\right],
$$

where $\lambda \in[-1,1]$. The corresponding pdf is given by

$$
\begin{aligned}
& f(x ; a, b, \lambda) \\
& \quad=a b x^{a-1}\left(1-x^{a}\right)^{b-1}\left[1-\lambda+2 \lambda\left(1-x^{a}\right)^{b}\right] .
\end{aligned}
$$

Ahmad et al. [19] proposed the transmuted Kumaraswamy (TKw) distribution as an extension of the Kw distribution and obtained the density and cumulative functions. However, they did not investigate an application to real data and explore the qf. In Figure 2, we plot the TKw density function for some parameter values.

The $r$ th moment of TKw can be obtained from (13) as

$$
\begin{array}{r}
E\left(X^{r}\right)=(1-\lambda) b B\left(1+\frac{r}{a}, b\right)+2 \lambda b B\left(1+\frac{r}{a}, 2 b\right), \\
r>-a,
\end{array}
$$

and the qf is given by

$$
\begin{aligned}
& F^{-1}(u ; a, b, \lambda) \\
& \quad=\left[1-\left(1-\frac{1+\lambda-\sqrt{(1+\lambda)^{2}-4 \lambda u}}{2 \lambda}\right)^{1 / b}\right]^{1 / a},
\end{aligned}
$$

\section{Extreme Values}

If $\bar{X}=n^{-1}\left(X_{1}+X_{2}+\cdots+X_{n}\right)$ denotes the sample mean from iid random variables following (2), then by standard central limit theorem $\sqrt{n}(\bar{X}-E(X)) / \sqrt{\operatorname{Var}(X)}$ converges in distribution to the standard normal as $n \rightarrow \infty$ under suitable conditions. However, one might be interested in the asymptotics of the extreme values $X_{\max }=\max \left(X_{1}, \ldots, X_{n}\right)$ and $X_{\min }=\min \left(X_{1}, \ldots, X_{n}\right)$. We consider the following:

(i) Suppose that $G$ belongs to the max. domain of attraction of the Gumbel extreme value distribution. Then, by Leadbetter et al. [20], there must be a strictly positive function, say $h(t)$, such that

$$
\lim _{t \rightarrow \infty} \frac{1-G(t+x h(t))}{1-G(t)}=\exp (-x),
$$

for every $x \in(-\infty, \infty)$. In our case, we have

$$
\begin{aligned}
\lim _{t \rightarrow \infty} & \frac{1-F(t+x h(t))}{1-F(t)} \\
= & \lim _{t \rightarrow \infty} \frac{(1-G(t+x h(t) ; \xi))(1-\lambda G(t+x h(t) ; \xi))}{(1-G(t ; \xi))(1-\lambda G(t ; \xi))} \\
= & \left(\lim _{t \rightarrow \infty} \frac{(1-G(t+x h(t) ; \xi))}{(1-G(t ; \xi))}\right) \\
& \cdot\left(\lim _{t \rightarrow \infty} \frac{(1-\lambda G(t ; \xi))}{(1-\lambda G(t ; \xi))}\right)=\lambda \exp (-x),
\end{aligned}
$$

for every $x \in(-\infty, \infty)$. Hence, it follows by Leadbetter et al. [20] that $F$ also belongs to the max. 


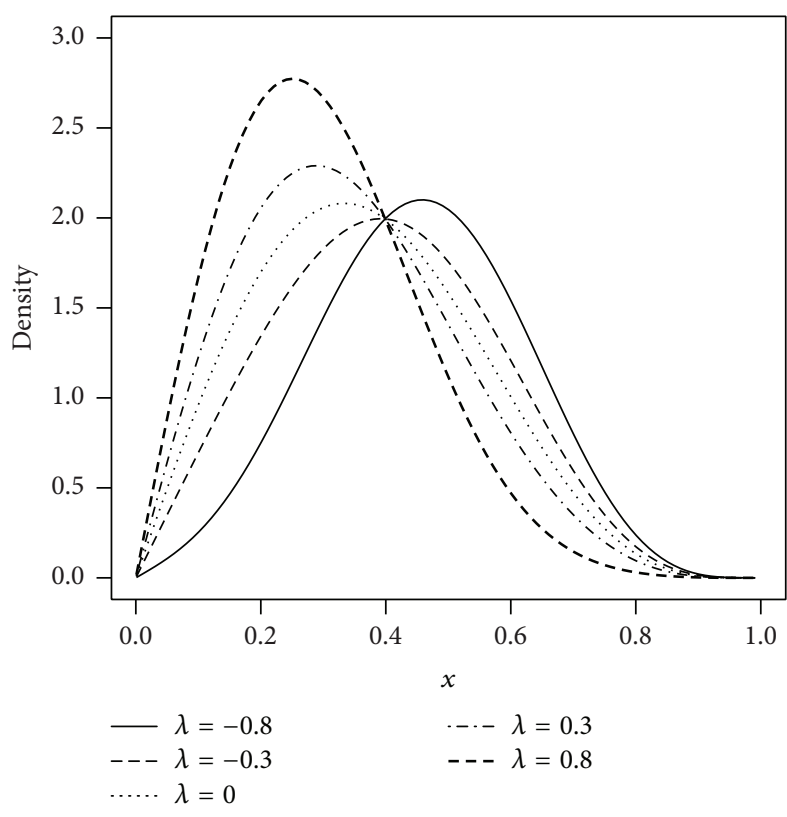

(a) $a=2, b=5$

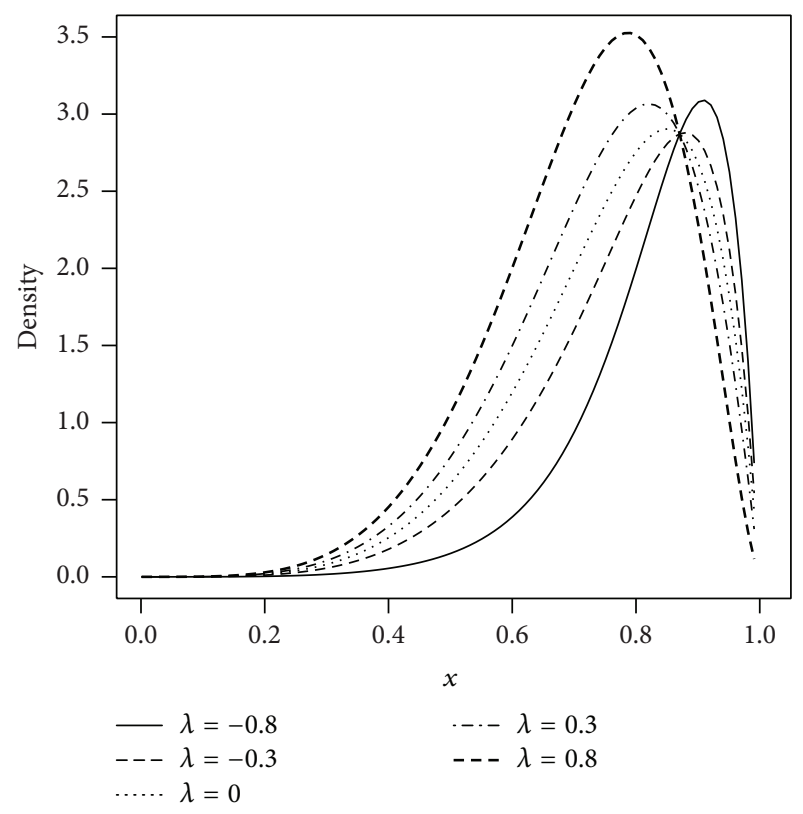

(b) $a=5, b=2$

FIGURE 2: Plots of the TKw density function for some parameter values.

domain of attraction of the Gumbel extreme value distribution with

$$
\lim _{n \rightarrow \infty} P\left(a_{n}\left(X_{\max }-b_{n}\right) \leq x\right)=\exp [-\lambda \exp (-x)]
$$

for some suitable norming constants $a_{n}>0$ and $b_{n}$.

(ii) Again, suppose that $G$ belongs to the max. domain of attraction of the Fréchet extreme value distribution. By Leadbetter et al. [20], there must exist a $\beta>0$, such that

$$
\lim _{t \rightarrow \infty} \frac{1-G(t x)}{1-G(t)}=x^{\beta}
$$

for every $x>0$. In our case,

$$
\begin{aligned}
& \lim _{t \rightarrow \infty} \frac{1-F(t x)}{1-F(t)} \\
& \quad=\left(\lim _{t \rightarrow \infty} \frac{1-G(t x ; \xi)}{1-G(t ; \xi)}\right)\left(\lim _{t \rightarrow \infty} \frac{1-\lambda G(t x ; \xi)}{1-\lambda G(t ; \xi)}\right) \\
& \quad=\lambda x^{\beta}
\end{aligned}
$$

for every $x>0$. Hence, it follows by Leadbetter et al. [20] that $F$ also belongs to the max. domain attraction of the Fréchet extreme value distribution with

$$
\lim _{n \rightarrow \infty} P\left(a_{n}\left(X_{\max }-b_{n}\right) \leq x\right)=\exp \left(-\lambda x^{\beta}\right)
$$

for some suitable norming constants $a_{n}>0$ and $b_{n}$.

(iii) Also, suppose that $G$ belongs to the max. domain of attraction of the Weibull extreme value distribution.
By Leadbetter et al. [20], there must be an $\alpha>0$ such that

$$
\lim _{t \rightarrow 0} \frac{1-G(t+x h(t))}{1-G(t)}=x^{\alpha}
$$

for every $x<0$. In our case, we have

$$
\begin{aligned}
\lim _{t \rightarrow 0} \frac{F(t x)}{F(t)} & =\lim _{t \rightarrow 0} \frac{(1+\lambda) G(t x ; \boldsymbol{\xi})-\lambda G^{2}(t x ; \boldsymbol{\xi})}{(1+\lambda) G(t ; \xi)-\lambda G^{2}(t ; \xi)} \\
& =x^{\alpha+1}
\end{aligned}
$$

for every $x<0$. Hence, it follows by Leadbetter et al. [20, Chapter 1] that $F$ also belongs to the max. domain of attraction of the Weibull extreme value distribution with

$$
\lim _{n \rightarrow \infty} P\left(a_{n}\left(X_{\max }-b_{n}\right) \leq x\right)=\exp \left[-(-x)^{\alpha+1}\right]
$$

for some suitable norming constants $a_{n}>0$ and $b_{n}$. Similar arguments apply to min. domains of attraction. That is, $F$ belongs to the same min. domain of attraction as that of $G$.

\section{Probability Weighted Moments}

The PWMs of a baseline model can be very useful to determine the moments of more complex distributions. Distributions that can be expressed in inverse form may present problems in estimating their parameters as functions of ordinary moments. For these distributions, the relations between the PWMs and the parameters have simpler analytical structure than those between the ordinary moments 
and the parameters. The PWMs are also widely used for estimating parameters of distributions from complete or censored samples.

We demonstrate that the $(n, s)$ th PWM of $X$, say $\delta_{n, s}=$ $E\left\{X^{n} F(X ; \xi, \lambda)^{s}\right\}$ (for $n, s=0,1, \ldots$ ), can be expressed as linear combinations of the baseline PWMs defined by $\tau_{n, s}=$ $\int_{-\infty}^{\infty} x^{n} G(X ; \xi)^{s} d x$. First, we can write $\tau_{n, s}$ from (20) and (23) by interchanging the sum and the integral

$$
\tau_{n, s}=\sum_{i=0}^{\infty} \frac{c_{n, i}}{i+s+1}
$$

Further, we have

$$
\delta_{n, r}=\int_{-\infty}^{+\infty} x^{n}\left[(1+\lambda) G(x ; \xi)-\lambda G(x ; \xi)^{2}\right] d x
$$

and then using the binomial expansion, we can express the PWMs of $X$ as

$$
\begin{aligned}
\delta_{n, r} & =\sum_{j=0}^{r}(-\lambda)^{j}(1+\lambda)^{r-j} \\
& \cdot\left(\begin{array}{l}
r \\
j
\end{array}\right)\left[(1+\lambda) \tau_{n, r-j}-2 \lambda \tau_{n, r+1-j}\right],
\end{aligned}
$$

where $\tau_{n, r-j}$ and $\tau_{n, r+1-j}$ are obtained from (44).

\section{Order Statistics}

Order statistics are required in many fields, such as climatology, engineering, and industry. Further, they play an important role in Statistical Inference and Nonparametric Statistics. In this section, we present some results with respect to the order statistics. We obtain an expression for the density of the $r$ th order statistic and the large sample distribution of the minimum and maximum when a random sample of size $n$ is drawn from the TG family. The density function of the $r$ th order statistic, say $X_{r: n}$, from a random sample of size $n$ drawn from (5) is given by

$$
\begin{aligned}
& f_{X_{r: n}}(x)=\frac{1}{B(r, n-r+1)} F(x)^{r-1}[1-F(x)]^{n-r} f(x) \\
& =\frac{1}{B(r, n-r+1)} \sum_{j=0}^{n-r}(-1)^{j} \\
& \quad \cdot\left(\begin{array}{c}
n-r \\
j
\end{array}\right)\left\{(1+\lambda) G(x ; \xi)-\lambda G(x ; \xi)^{2}\right\}^{r-1+j}[1 \\
& \quad+\lambda-2 \lambda G(x ; \xi)] g(x ; \xi) .
\end{aligned}
$$

The $k$ th order moment of $X_{r: n}$ is obtained from (20) as

$$
\begin{gathered}
E\left(X_{r: n}^{k}\right)=\frac{1}{B(r, n-r+1)} \sum_{j=0}^{n-r}(-1)^{j}\left(\begin{array}{c}
n-r \\
j
\end{array}\right) \\
\cdot \int_{0}^{\infty} x^{k}\left\{(1+\lambda) G(x ; \xi)-\lambda G(x ; \xi)^{2}\right\}^{r+j-1} \\
\cdot[1+\lambda-2 \lambda G(x ; \xi)] g(x ; \xi) d x \\
=\frac{1}{B(r, n-r+1)} \sum_{j=0}^{n-r}(-1)^{j}\left(\begin{array}{c}
n-r \\
j
\end{array}\right) J,
\end{gathered}
$$

where $J=\int_{0}^{1} G^{-1}(t ; \xi)(1+\lambda-2 \lambda t)\left[(1+\lambda) t-\lambda t^{2}\right]^{r+j-1} d t$ can be evaluated numerically for most parent distributions using statistical software.

\section{Information Theory}

11.1. Entropies. An entropy is a measure of variation or uncertainty of a random variable $X$. Two well-known entropy measures are the Rényi and Shannon entropies. The Rényi entropy of a random variable $X$ with pdf $f(x)$ is defined by

$$
I_{R}(\rho)=\frac{1}{(1-\rho)} \log \left(\int_{0}^{\infty} f^{\rho}(x) d x\right)
$$

for $\rho>1$ and $\rho \neq 1$. The Rényi entropy for the TG family should be evaluated numerically.

The Shannon entropy of a random variable $X$ is defined by $E\{-\log [f(X)]\}$. It is the special case of the Rényi entropy when $\rho \rightarrow 1$. For the proposed model in (1), the Shannon entropy reduces to

$$
\begin{aligned}
E\{-\log [f(X)]\}= & -E\{\log [g(X ; \xi)]\} \\
& -E\{\log [1+\lambda-2 \lambda G(X ; \xi)]\} \\
= & -\left(I_{1}+I_{2}\right),
\end{aligned}
$$

where

$$
\begin{aligned}
I_{1} & =E\{\log [g(X ; \xi)]\} \\
& =\int_{0}^{\infty} \log [g(x ; \xi)][1+\lambda-2 \lambda G(x ; \xi)] g(x ; \xi) d x \\
& =\frac{1}{2 \lambda} \int_{1-\lambda}^{1+\lambda} t \log \left\{g\left[G^{-1}\left(\frac{1+\lambda-t}{2 \lambda} ; \xi\right)\right]\right\} d t \\
& =A_{1}(\lambda ; \xi), \\
I_{2} & =E[\log (1+\lambda-2 \lambda G(X ; \xi))]=\frac{1}{2}\left[(1+\lambda)^{2}\right. \\
& \left.\cdot \log (1+\lambda)-(\lambda-1)^{2} \log (1-\lambda)-2 \lambda\right] .
\end{aligned}
$$


By substituting the last two expressions in (50), the Shannon entropy becomes

$$
\begin{aligned}
& E\{-\log [f(X)]\}=-A_{1}(\lambda ; \xi) \\
& \quad-\frac{1}{2}\left[(1+\lambda)^{2} \log (1+\lambda)-(\lambda-1)^{2} \log (1-\lambda)\right. \\
& \quad-2 \lambda] .
\end{aligned}
$$

Next, we consider the Rényi entropy. From (1), we have

$$
\begin{aligned}
\int_{0}^{\infty} & f^{\rho}(x) d x \\
\quad & \frac{1}{2 \lambda} \int_{1-\lambda}^{1+\lambda} t^{\rho+1}\left\{g\left[G^{-1}\left(\frac{1+\lambda-t}{2 \lambda} ; \xi\right)\right]\right\}^{\rho} d t \\
& =A_{2}(\lambda ; \xi)
\end{aligned}
$$

which can be computed numerically.

11.2. Kullback-Leibler (KL) Divergence. Consider two distributions from the same family (but with different parametric configuration). To be more specific, let $P(x) \sim T G\left(\lambda_{1}, \xi_{1}\right)$ and $Q(x) \sim T G\left(\lambda_{2}, \xi_{2}\right)$. Then, the KL divergence measure between $P(x)$ and $Q(x)$, say $\mathrm{KL}(P \mid Q)$, is given by

$$
\begin{aligned}
& \mathrm{KL}(P \mid Q)=\int P(x) \log \left(\frac{P(x)}{Q(x)}\right) d x=\int P(x) \\
& \cdot \log \left(\frac{\left[1+\lambda_{1}-2 \lambda_{1} G\left(x ; \xi_{1}\right)\right] g\left(x ; \xi_{1}\right)}{\left[1+\lambda_{2}-2 \lambda_{2} G\left(x ; \xi_{2}\right)\right] g\left(x ; \xi_{2}\right)}\right) d x \\
& =\int P(x) \log \left(\left[1+\lambda_{1}-2 \lambda_{1} G\left(x ; \xi_{1}\right)\right]\right) d x \\
& +\int P(x) \log \left(g\left(x ; \xi_{1}\right)\right) d x-\int P(x) \\
& \quad \cdot \log \left(\left[1+\lambda_{2}-2 \lambda_{2} G\left(x ; \xi_{2}\right)\right]\right) d x-\int P(x) \\
& \cdot \log \left(g\left(x ; \xi_{2}\right)\right) d x=J_{1}+J_{2}-J_{3}-J_{4},
\end{aligned}
$$

where

$$
\begin{aligned}
J_{1}= & \int P(x) \log \left(\left[1+\lambda_{1}-2 \lambda_{1} G\left(x ; \xi_{1}\right)\right]\right) d x \\
= & \frac{1}{2}\left[-2 \lambda_{1}-\left(\lambda_{1}-1\right)^{2} \log \left(1-\lambda_{1}\right)+\left(1+\lambda_{1}\right)^{2}\right. \\
& \left.\cdot \log \left(1+\lambda_{1}\right)\right] \\
J_{2}= & A_{1}(\lambda ; \xi), \\
J_{3}= & \frac{1}{2 \lambda_{1}} \int_{1-\lambda_{1}}^{1+\lambda_{1}} t \log \left\{1+\lambda_{2}\right. \\
& \left.-2 \lambda_{2} G\left[G^{-1}\left(\frac{1+\lambda_{1}-t}{2 \lambda_{1}} ; \xi_{2}\right)\right] ; \xi_{1}\right\} d t .
\end{aligned}
$$

Special Case. If $\boldsymbol{\xi}_{1}=\boldsymbol{\xi}_{2}$, then $J_{3}$ reduces to

$$
\begin{aligned}
A_{3} & (\lambda ; \xi)=\int_{1-\lambda_{1}}^{1+\lambda_{1}} t \\
& \cdot \log \left\{1+\lambda_{2}-2 \lambda_{2} G\left[G^{-1}\left(\frac{1+\lambda_{1}-t}{2 \lambda_{1}} ; \xi_{2}\right)\right]\right\} d t \\
& =\left(4 \lambda_{1}^{2} \lambda_{2}^{2}\right)^{-1}\left[-\lambda_{1} \lambda_{2}\left(1+\lambda_{1}\right)\right]\left[-2+\left(-2+3 \lambda_{1}\right.\right. \\
& \left.\left.+3 \lambda_{1}^{2}\right) \lambda_{2}\right]-\left(4 \lambda_{1}^{2} \lambda_{2}^{2}\right)^{-1}\left[\lambda_{1} \lambda_{2}\left(\lambda_{1}-1\right)(-2\right. \\
& \left.\left.+\left(-2+3 \lambda_{1}+\lambda_{1}^{2}\right) \lambda_{2}\right)\right]+2\left(4 \lambda_{1}^{2} \lambda_{2}^{2}\right)^{-1}\left(1+\lambda_{2}\right) \\
& \cdot\left(-1+\left(-1+2 \lambda_{1}+2 \lambda_{1}^{2}\right) \lambda_{2}\right) \log \left(1+\lambda_{2}\right)-2[1 \\
& \left.-2\left(1+\lambda_{1}+\lambda_{1}^{2}\right) \lambda_{2}+\lambda_{2}^{2}\left(1-2 \lambda_{1}-2 \lambda_{1}^{2}+4 \lambda_{1}^{3}\right)\right] \\
& \cdot \log \left[1+\lambda_{2}-2 \lambda_{1}^{2} \lambda_{2}\right] .
\end{aligned}
$$

Thus, $J_{3}=\left(1 / 2 \lambda_{1}\right) A_{3}(\lambda ; \xi)$. Also, in this case, $J_{2}=J_{4}$. Hence, the KL divergence is given by

$$
\begin{aligned}
& \mathrm{KL}(P \mid Q)=\frac{1}{2}\left[-2 \lambda_{1}-\left(\lambda_{1}-1\right)^{2} \log \left(1-\lambda_{1}\right)\right. \\
& \left.+\left(1+\lambda_{1}\right)^{2} \log \left(1+\lambda_{1}\right)\right]-2 A_{1}(\lambda ; \xi)-\frac{1}{2 \lambda_{1}} \\
& \cdot A_{3}(\lambda ; \xi) .
\end{aligned}
$$

\section{Bivariate and Multivariate Generalization}

First, we consider a bivariate extension of the new model. The joint cdf is expressed as

$$
\begin{array}{r}
F(x, y ; \xi, \lambda)=(1+\lambda) G(x, y ; \xi)-\lambda[G(x, y ; \xi)]^{2}, \\
(x, y) \in \mathscr{D} \subseteq \mathbb{R}, \lambda \in[-1,1],
\end{array}
$$

where $G(x, y ; \xi)$ is a bivariate continuous distribution with marginal cdfs $G_{1}(x ; \xi)$ and $G_{2}(x ; \xi)$.

The corresponding joint pdf is given by

$$
\begin{aligned}
f(x, y)= & \frac{\partial^{2}}{\partial x \partial y} F(x, y) \\
= & {[\lambda+G(x, y)] \frac{\partial^{2}}{\partial x \partial y} G(x, y) } \\
& -2 \lambda\left[\frac{\partial}{\partial x} G(x, y)\right]\left[\frac{\partial}{\partial y} G(x, y)\right] .
\end{aligned}
$$

The marginal cdfs are given by

(i) $F(x)=(1+\lambda) G_{1}(x)-\lambda G_{1}(x)^{2}$,

(ii) $F(y)=(1+\lambda) G_{2}(y)-\lambda G_{2}(y)^{2}$.

The marginal pdfs are given by

(i) $f(x)=(1+\lambda) g_{1}(x)-2 \lambda G_{1}(x) g_{1}(x)$,

(ii) $f(y)=(1+\lambda) g_{2}(y)-2 \lambda G_{2}(y) g_{2}(y)$. 
A natural multivariate extension (say, $m$-variate, $m \geq 2$ ) of the above bivariate (for $\lambda \in[-1,1]$ ) is given by

$$
\begin{aligned}
& F\left(x_{1}, \ldots, x_{m} ; \xi, \lambda\right)=(1+\lambda) G\left(x_{1}, \ldots, x_{m} ; \xi\right) \\
&-\lambda\left[G\left(x_{1}, \ldots, x_{m} ; \xi\right)\right]^{2}, \\
&\left(x_{1}, \ldots, x_{m}\right) \in \mathscr{D} \subseteq \mathbb{R}
\end{aligned}
$$

where $G\left(x_{1}, \ldots, x_{m} ; \xi\right)$ is a multivariate continuous distribution with marginal cdfs $G_{i}\left(x_{i} ; \xi\right)$, for $i=1,2, \ldots, m$.

\section{Maximum Likelihood Estimation}

Several methods for parameter estimation have been proposed in the literature but the maximum likelihood method is the most commonly employed. The maximum likelihood estimators (MLEs) enjoy desirable properties and can be used to obtain confidence intervals for the model parameters. In this section, we consider the estimation of the unknown parameters of the $T G$ family from complete samples only by maximum likelihood. Let $x_{1}, \ldots, x_{n}$ be observed values from this family with parameters $\lambda$ and $\xi$.

Let $\boldsymbol{\theta}=\left(\lambda, \xi^{\top}\right)^{\top}$ be the $p \times 1$ parameter vector. The total $\log$-likelihood function for $\boldsymbol{\theta}$ is given by

$$
\begin{aligned}
\ell(\boldsymbol{\theta})= & \sum_{i=1}^{n} \log \left[g\left(x_{i} ; \boldsymbol{\xi}\right)\right] \\
& +\sum_{i=1}^{n} \log \left[1+\lambda-2 \lambda G\left(x_{i} ; \xi\right)\right] .
\end{aligned}
$$

The components of the score function $U(\boldsymbol{\theta})=\left(U_{\lambda}, U_{\xi}\right)^{\top}$ are $($ for $k=1, \ldots, p)$

$$
\begin{aligned}
U_{\lambda}= & -2 \sum_{i=1}^{n}\left[\frac{G\left(x_{i} ; \xi\right)}{1+\lambda-2 \lambda G\left(x_{i} ; \xi\right)}\right], \\
U_{\xi_{k}}= & \sum_{i=1}^{n}\left[\frac{\partial g\left(x_{i} ; \xi\right) / \partial \xi_{k}}{g\left(x_{i} ; \xi\right)}\right] \\
& -2 \lambda \sum_{i=1}^{n}\left[\frac{\partial G\left(x_{i} ; \xi\right) / \partial \xi_{k}}{1+\lambda-2 \lambda G\left(x_{i} ; \xi\right)}\right] .
\end{aligned}
$$

We can obtain the MLE $\widehat{\boldsymbol{\theta}}=\left(\widehat{\lambda}, \widehat{\boldsymbol{\xi}}^{\top}\right)^{\top}$ of $\boldsymbol{\theta}=\left(\lambda, \boldsymbol{\xi}^{\top}\right)^{\top}$ by maximizing the log-likelihood either directly by using the $\mathrm{R}$ (optim function), SAS (PROC NLMIXED), and Ox (subroutine MaxBFGS) programs or by setting $U_{\lambda}$ and $U_{\xi}$ equal to zero and solving the equations simultaneously. These equations can be solved numerically using iterative methods such as the Newton-Raphson type algorithms.

For interval estimation on the model parameters, we require the observed information matrix

$$
J(\boldsymbol{\theta})=-\left(\begin{array}{ccc}
U_{\lambda \lambda} & \mid & U_{\lambda \xi}^{\top} \\
-- & -- & -- \\
U_{\lambda \xi} & \mid & U_{\xi \xi}
\end{array}\right),
$$

TABle 3: Descriptive statistics for the data set.

\begin{tabular}{cccccccc}
\hline$n$ & Min. & $Q_{1}$ & $Q_{2}$ & Mean & $Q_{3}$ & Max. & Var. \\
\hline 166 & 0.0100 & 0.0100 & 0.0300 & 0.0912 & 0.1300 & 0.6900 & 0.0176 \\
\hline
\end{tabular}

whose elements are

$$
\begin{aligned}
U_{\lambda \lambda}= & -2 \sum_{i=1}^{n} \frac{G\left(x_{i} ; \xi\right)^{2}}{\left[1+\lambda-2 \lambda G\left(x_{i} ; \xi\right)\right]^{2}}, \\
U_{\lambda \xi_{k}}= & -2(1+\lambda) \sum_{i=1}^{n} \frac{\partial G\left(x_{i} ; \xi\right) / \partial \xi_{k}}{\left[1+\lambda-2 \lambda G\left(x_{i} ; \xi\right)\right]^{2}}, \\
U_{\xi_{k} \xi_{l}=} & \sum_{i=1}^{n} \frac{g_{k l}^{\prime \prime}\left(x_{i} ; \xi\right) g\left(x_{i} ; \xi\right)-g_{k}^{\prime}\left(x_{i} ; \xi\right) g_{l}^{\prime}\left(x_{i} ; \xi\right)}{g^{2}\left(x_{i} ; \xi\right)} \\
& -2 \lambda \sum_{i=1}^{n} \frac{G_{k l}^{\prime \prime}\left(x_{i} ; \xi\right)}{\left[1+\lambda-2 \lambda G\left(x_{i} ; \xi\right)\right]} \\
& -4 \lambda \sum_{i=1}^{n} \frac{G_{k}^{\prime}\left(x_{i} ; \xi\right) G_{l}^{\prime}\left(x_{i} ; \xi\right)}{\left[1+\lambda-2 \lambda G\left(x_{i} ; \xi\right)\right]^{2}},
\end{aligned}
$$

where $t_{k}^{\prime}(\cdot ; \xi)=\partial t(\cdot ; \xi) / \partial \xi_{k}$ and $t_{k l}^{\prime \prime}(\cdot ; \xi)=\partial^{2} t(\cdot ; \xi) / \partial \xi_{k} \partial \xi_{l}$.

We can easily check if the fit using the $T G$ model is statistically "superior" to a fit using the $G$ model by testing the null hypothesis $H_{0}: \lambda=0$ against $H_{1}: \lambda \neq 0$. For testing $H_{0}: \lambda=0$, the likelihood ratio (LR) statistic is given by $w=2\{\ell(\widehat{\lambda}, \widehat{\xi})-\ell(0, \widetilde{\xi})\}$, where $\hat{\lambda}$ and $\widehat{\xi}$ and $\widetilde{\xi}$ are the unrestricted and restricted estimates obtained from the maximization of $\ell(\boldsymbol{\theta})$ under $H_{1}$ and $H_{0}$, respectively. The limiting distribution of this statistic is $\chi_{1}^{2}$ under the null hypothesis. The null hypothesis is rejected if the test statistic exceeds the upper $100(1-\gamma) \%$ quantile of the $\chi_{1}^{2}$ distribution.

\section{An Application}

In this section, we compare the results of fitting the TKw and Kw distributions to a real data set. We estimate the unknown parameters of the models by the maximum likelihood (as discussed in Section 13) and all the computations are performed using the NLMixed subroutine of the SAS software. The data are from a study on anxiety performed in a group of 166 "normal" women, that is, outside of a pathological clinical picture (Townsville, Queensland, Australia). The data originally reported by Smithson and Verkuilen [21] are as follows: $0.01,0.17,0.01,0.05,0.09,0.41,0.05,0.01,0.13,0.01$, $0.05,0.17,0.01,0.09,0.01,0.05,0.09,0.09,0.05,0.01,0.01,0.01$, $0.29,0.01,0.01,0.01,0.01,0.01,0.01,0.01,0.01,0.09,0.37,0.05$, $0.01,0.05,0.29,0.09,0.01,0.25,0.01,0.09,0.01,0.05,0.21,0.01$, $0.01,0.01,0.13,0.17,0.37,0.01,0.01,0.09,0.57,0.01,0.01,0.13$, $0.05,0.01,0.01,0.01,0.01,0.09,0.13,0.01,0.01,0.09,0.09,0.37$, $0.01,0.05,0.01,0.01,0.13,0.01,0.57,0.01,0.01,0.09,0.01,0.01$, $0.01,0.01,0.01,0.01,0.05,0.01,0.01,0.01,0.13,0.01,0.25,0.01$, $0.01,0.09,0.13,0.01,0.01,0.05,0.13,0.01,0.09,0.01,0.05,0.01$, $0.05,0.01,0.09,0.01,0.37,0.25,0.05,0.05,0.25,0.05,0.05,0.01$, $0.05,0.01,0.01,0.01,0.17,0.29,0.57,0.01,0.05,0.01,0.09,0.01$, $0.09,0.49,0.45,0.01,0.01,0.01,0.05,0.01,0.17,0.01,0.13,0.01$, 
TABLE 4: Estimates of the parameters (standard errors in parentheses), goodness-of-fit statistics, and confidence intervals with significance level at $5 \%$ for the data set.

\begin{tabular}{|c|c|c|c|c|c|}
\hline Model & Estimates & $\begin{array}{c}\text { Confidence } \\
\text { intervals }\end{array}$ & AIC & BIC & CAIC \\
\hline $\mathrm{TKw}$ & $\begin{array}{l}\widehat{a}=0.7037(0.0483) \\
\widehat{b}=3.8089(0.6890) \\
\widehat{\lambda}=0.5680(0.1784)\end{array}$ & $\begin{array}{l}(0.6083,0.7991) \\
(2.4485,5.1693) \\
(0.2159,0.9202)\end{array}$ & -489.8 & -480.5 & -489.7 \\
\hline $\mathrm{Kw}$ & $\begin{array}{l}\widehat{a}=0.6425(0.0457) \\
\widehat{b}=4.4733(0.5827)\end{array}$ & $\begin{array}{l}(0.5523,0.7326) \\
(3.3228,5.6239)\end{array}$ & -484.6 & -478.4 & -484.6 \\
\hline
\end{tabular}
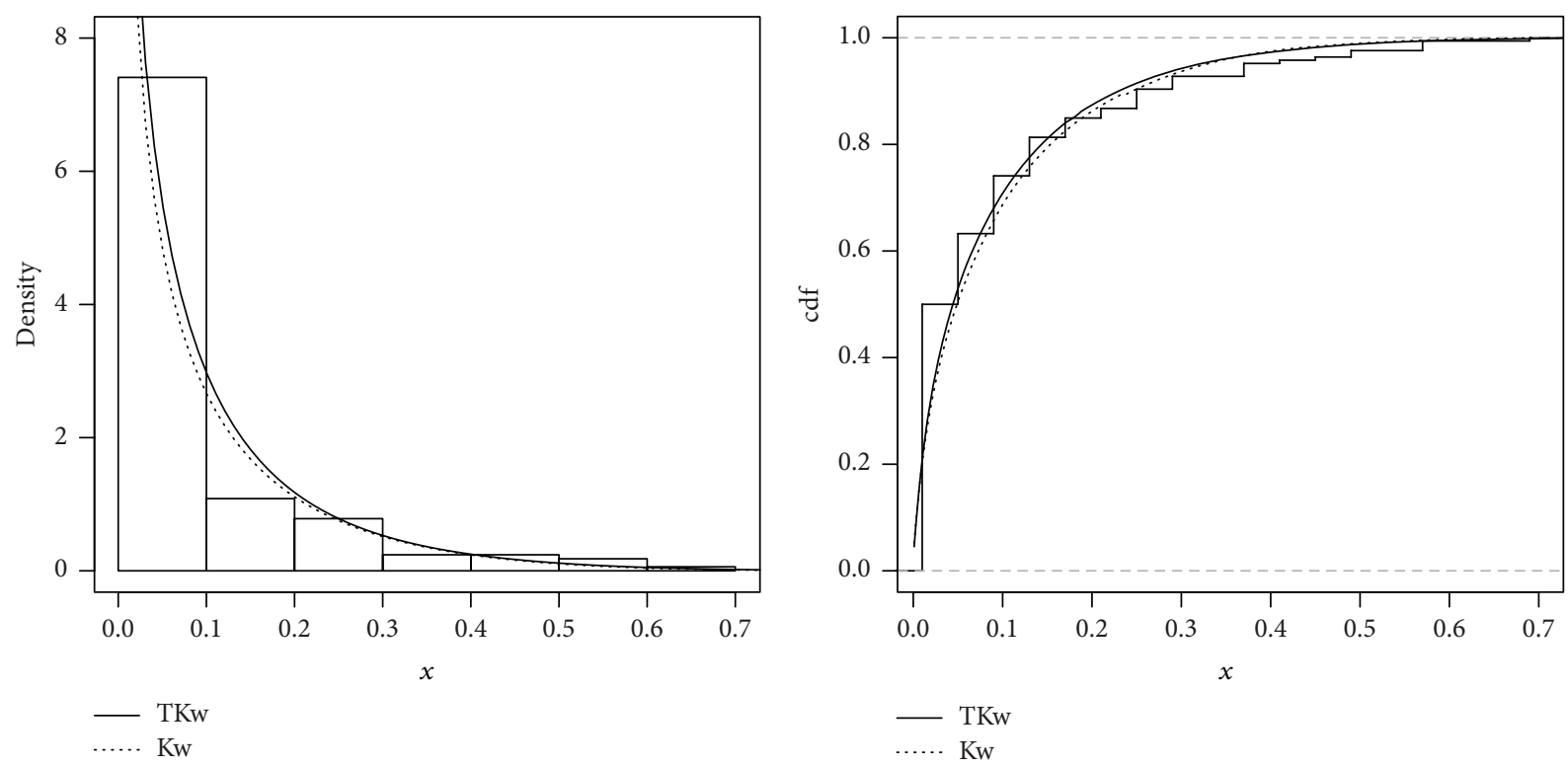

FIgURE 3: Estimated pdf and cdf from the fitted TKw and Kw models for the data.

$0.21,0.13,0.01,0.01,0.17,0.01,0.01,0.21,0.13,0.69,0.25,0.01$, $0.01,0.09,0.13,0.01,0.05,0.01,0.01,0.29,0.25,0.49,0.01$, and 0.01 .

Table 3 lists some descriptive statistics of these data. Table 4 gives the MLEs (with corresponding standard errors in parentheses) and some goodness of fit measures for the fitted TKw and $\mathrm{Kw}$ distributions. Since the values of the Akaike information criterion (AIC), Bayesian information criterion (BIC), and consistent Akaike information criterion (CAIC) are smaller for the TKw distribution compared to those values of the $\mathrm{Kw}$ model, the new distribution is a very competitive model to these data.

In order to test the null hypothesis $H_{0}: \lambda=0$ against the alternative hypothesis $H_{1}: \lambda \neq 0$, we obtain a confidence interval (with $5 \%$ significance level). The $95 \%$ confidence interval for $\lambda$ is $(0.2159,0.9202)$ and since it does not contain zero, we can reject the null hypothesis in favor of the alternative hypothesis that the data set is generated from a TKw model with $\lambda \neq 0$. Further, the LR statistic (see Section 13) to test $H_{0}: \mathrm{Kw}$ against $H_{1}$ : TKw is 7.2 ( $p$ value $<0.01)$. Thus, using any usual significance level, we reject the null hypothesis in favor of the TKw distribution, that is, this distribution is significantly better than the $\mathrm{Kw}$ distribution to explain the current data.
Plots of the pdf and cdf of the fitted TKw and Kw models to these data are displayed in Figure 3. They indicate that the $\mathrm{TKw}$ model is superior to the Kw model in terms of model fitting.

\section{Conclusions}

In this paper, we explore general properties of the transmuted- $G$ family of distributions. This family is obtained by adding shape parameters to an existing well-known distribution by using the transmutation map approach [1]. We investigate some of its general mathematical properties including ordinary and incomplete moments, generating function, probability weighted moments, Shannon and Rényi entropies, Kullback-Leibler divergence, and limiting behavior of the extremum values. The existing literature does not include these general properties. We also provide a real life application to prove empirically the usefulness of the transmuted-G family. The application indicates that the transmuted model performs better compared to the parent model. Consequently, it merits further a thorough study in terms of its extension to a bivariate and subsequently multivariate set-up (we have discussed briefly this topic in Section 12). Needless to say, inferential procedures, especially 
under the Bayesian paradigm, will not be an easy task. We plan to work on it as a future project and will report our findings elsewhere.

\section{Conflict of Interests}

The authors declare that there is no conflict of interests regarding the publication of this paper.

\section{References}

[1] W. Shaw and I. Buckley, "The alchemy of probability distributions: beyond Gram-Charlier expansions, and a skew-kurtoticnormal distribution from a rank transmutation map," Research Report, 2007.

[2] G. R. Aryal and C. P. Tsokos, "On the transmuted extreme value distribution with application," Nonlinear Analysis: Theory, Methods \& Applications, vol. 71, no. 12, pp. e1401-e1407, 2009.

[3] G. R. Aryal and C. P. Tsokos, "Transmuted Weibull distribution: a generalization of the Weibull probability distribution," European Journal of Pure and Applied Mathematics, vol. 4, no. 2, pp. 89-102, 2011.

[4] G. R. Aryal, "Transmuted log-logistic distribution," Journal of Statistics Applications \& Probability, vol. 2, no. 1, pp. 11-20, 2013.

[5] M. S. Khan and R. King, "Transmuted modified weibull distribution: a generalization of the modified weibull probability distribution," European Journal of Pure and Applied Mathematics, vol. 6, no. 1, pp. 66-88, 2013.

[6] I. Elbatal, "Transmuted modified inverse Weibull distribution: a generalization of the modified inverse Weibull probability distribution," International Journal of Mathematical Archive, vol. 4, no. 8, pp. 117-129, 2013.

[7] I. Elbatal and G. R. Aryal, "On the transmuted additive Weibull distribution," Austrian Journal of Statistics, vol. 42, no. 2, pp. 117132, 2013.

[8] G. S. Mudholkar and D. K. Srivastava, "Exponentiated Weibull family for analyzing bathtub failure-rate data," IEEE Transactions on Reliability, vol. 42, no. 2, pp. 299-302, 1993.

[9] R. C. Gupta, P. L. Gupta, and R. D. Gupta, "Modeling failure time data by Lehman alternatives," Communications in Statistics. Theory and Methods, vol. 27, no. 4, pp. 887-904, 1998.

[10] R. D. Gupta and D. Kundu, "Generalized exponential distributions," Australian \& New Zealand Journal of Statistics, vol. 41, no. 2, pp. 173-188, 1999.

[11] S. Nadarajah, "The exponentiated Gumbel distribution with climate application," Environmetrics, vol. 17, no. 1, pp. 13-23, 2006.

[12] C. S. Kakde and D. T. Shirke, "On exponentiated lognormal distribution," International Journal of Agricultural and Statistical Sciences, vol. 2, pp. 319-326, 2006.

[13] S. Nadarajah and A. K. Gupta, "The exponentiated gamma distribution with application to drought data," Calcutta Statistical Association Bulletin, vol. 59, no. 233-234, pp. 29-54, 2007.

[14] F. Merovci, "Transmuted Lindley distribution," International Journal of Open Problems in Computer Science and Mathematics, vol. 6, no. 2, pp. 63-72, 2013.

[15] M. R. Mahmoud and R. M. Mandouh, "On the transmuted Fréchet distribution," Journal of Applied Sciences Research, vol. 9, no. 10, pp. 5553-5561, 2013.

[16] F. Merovci and L. Puka, "Transmuted Pareto distribution," ProbStat Forum, vol. 7, pp. 1-11, 2014.
[17] I. S. Gradshteyn and I. M. Ryzhik, Table of Integrals, Series, and Products, Academic Press, New York, NY, USA, 2000.

[18] S. K. Ashour and M. A. Eltehiwy, "Transmuted lomax distribution," American Journal of Applied Mathematics and Statistics, vol. 1, no. 6, pp. 121-127, 2013.

[19] A. Ahmad, S. P. Ahmad, and A. Ahmed, "Characterization and estimation of transmuted Kumaraswamy distribution," 9, vol. 5, no. 9, pp. 168-174, 2015.

[20] M. R. Leadbetter, G. Lindgren, and H. Rootzn, Extremes and Related Properties of Random Sequences and Processes, Springer, New York, NY, USA, 1987.

[21] M. Smithson and J. Verkuilen, "A better lemon squeezer? Maximum-likelihood regression with beta-distributed dependent variables," Psychological Methods, vol. 11, no. 1, pp. 54-71, 2006. 


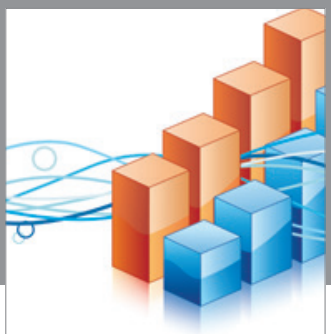

Advances in

Operations Research

vatem alat4

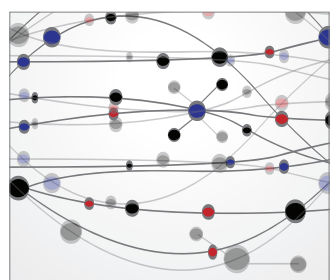

\section{The Scientific} World Journal
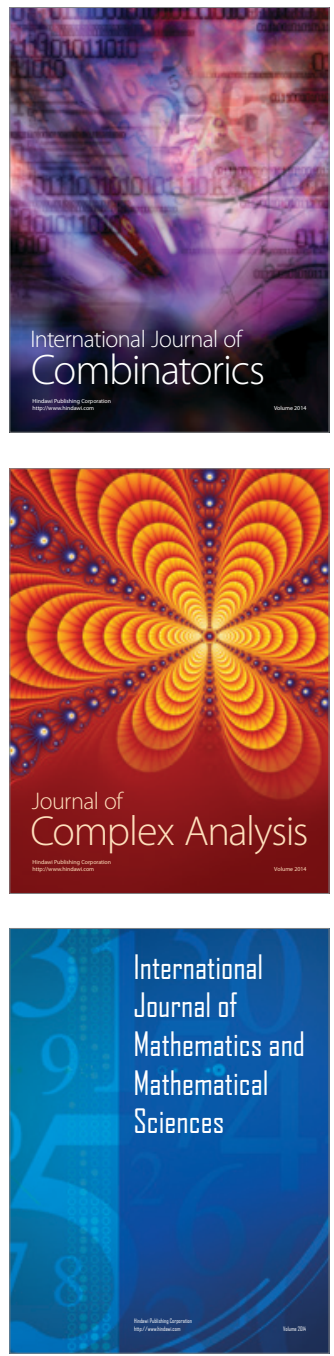
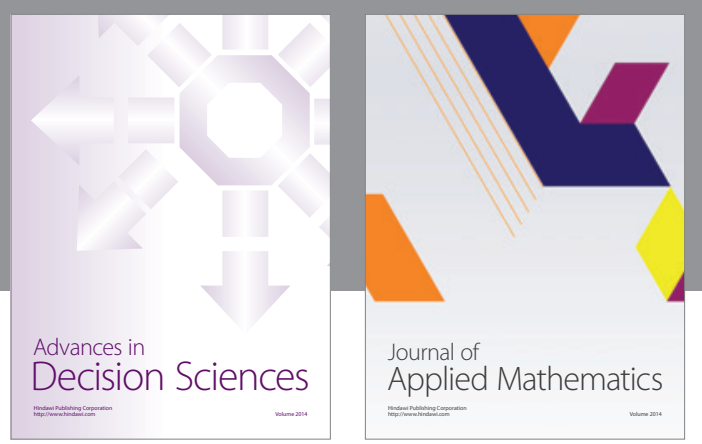

Algebra

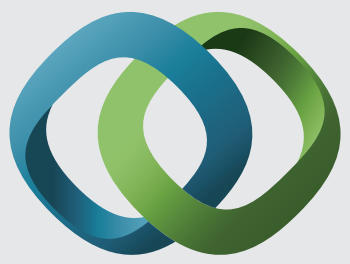

\section{Hindawi}

Submit your manuscripts at

http://www.hindawi.com
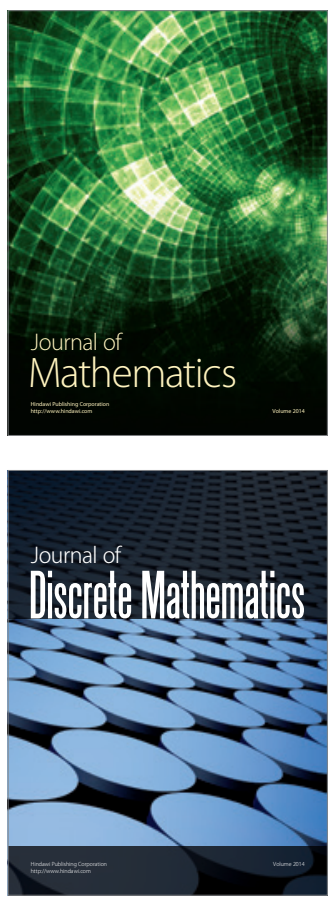

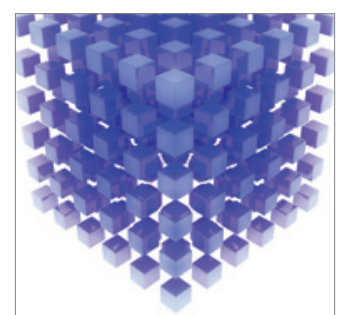

Mathematical Problems in Engineering
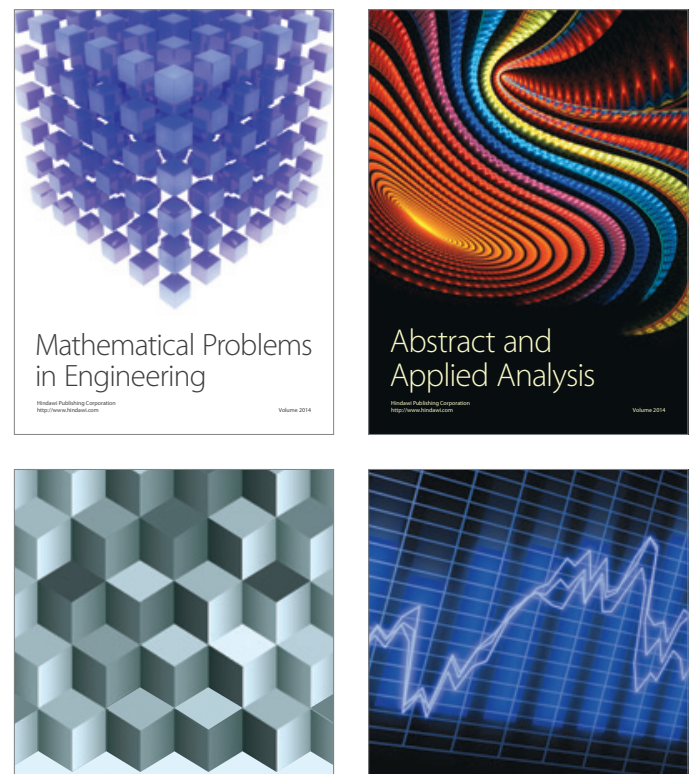

Journal of

Function Spaces

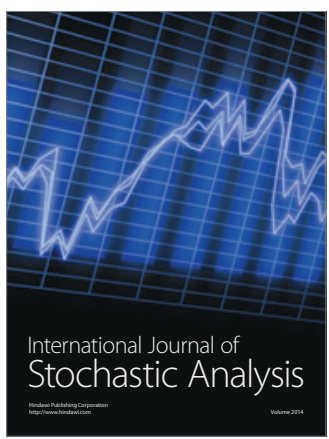

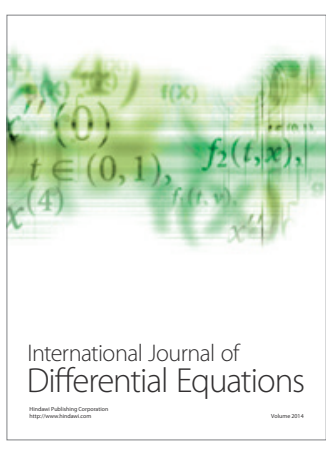
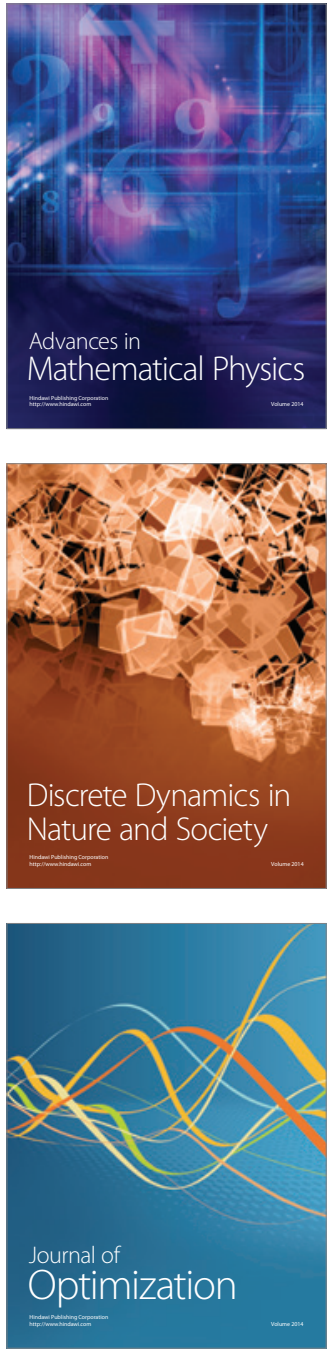